Capítulo 10

\title{
Gynerium sagittatum, una especie para la innovación y el desarrollo en el Departamento de Sucre
}

\author{
Gynerium sagittatum, a species for innovation and development in the \\ department of Sucre
}

\author{
Pedro Arturo Martínez Osorio ${ }^{1}$ \\ Paula Da Cruz Landim² \\ Tomás Queiroz Ferreira Barata ${ }^{3}$
}

\section{Introducción}

En la actualidad muchos de los problemas ambientales que ocurren alrededor del mundo han contribuido a incrementar un interés por buscar alternativas para generar procesos, productos y servicios sostenibles, preocupaciones que, desde la mirada del diseño, pueden incluirse dentro de conceptos como Life Cycle Design y Design para la sustentabilidad (Manzini \& Vezzoli, 2002).

1 UNESP, Universidade Estadual Paulista Júlio de Mesquita Filho, Bauru, Brasil. Arquitecto. Candidato a Doctor en Design. Maestría en Educación. Miembro del grupo de investigación NUPECAM, FAAC, UNESP, miembro del grupo de investigación Teoría e Historia de la Arquitectura y la Ciudad, CECAR, Sincelejo. Investigador Junior de Colciencias. Email:pedro.martinez@cecar.edu.co ORCID iD: https://orcid.org/0000-0002-9024$\underline{0918}$

2 UNESP, Universidade Estadual Paulista Júlio de Mesquita Filho, Bauru, Brasil. Arquitecta. Doctora en Arquitectura y Urbanismo, Maestría en Geografía. Profesora Libre docente en Design de producto FAAC, UNESP - campus de Bauru, Brasil. Coordinadora del Programa de Posgrado en Design de la Facultad de Arquitectura, Artes y Comunicación - FAAC, UNESP. Directora del grupo de investigación NUPECAM, FAAC, UNESP. Email: paula@faac.unesp.br ORCID iD: https://orcid.org/0000-0002-1510-7738

3 UNESP, Universidade Estadual Paulista Júlio de Mesquita Filho, Bauru, Brasil. Arquitecto y urbanista. Doctor en Ingeniería Civil, Maestría en Arquitectura y Urbanismo. Director del Departamento de Design de la Facultad de Arquitectura, Artes y Comunicación - FAAC, UNESP. Profesor asistente, Departamento de Design - FAAC, UNESP. Email: barata@faac.unesp.br ORCID iD: http://orcid.org/0000-0002-1573-5590 
Nuevas perspectivas de utilización de las llamadas "nuevas maderas", como algunos árboles frutales, coco, caucho, bambú, rattan, palma, otras fibras y residuos agrícolas, rápidamente han ganado importancia en el mercado, a partir de la experimentación e Innovación (Durst et al., 2004; Pereira \& Beraldo, 2008). Estos intereses de investigación se consolidan, cada vez más, como uno de los escenarios de Innovación y Desarrollo donde ocurre un fuerte y dinámico debate en la búsqueda de materiales y procesos alternativos como reemplazo a la producción tradicional de la madera.

La especie Gynerium sagittatum (Aubl.) P.Beauv. (Figura 12), Wild cane, llamada en Colombia como "Caña Flecha", es denominada en América del Sur con muchos nombres, siendo el más común "Caña brava". En Brasil es conocida también por muchos nombres populares, los más conocidos "Cana-brava" y "Cana-do-rio" (Coradin et al., 2011, p. 254).

Gynerium sagittatum es una especie de hierba leñosa de la familia Poaceae, cuya reproducción se da generalmente en los bancos de arena a orilla de los ríos; crece, en promedio, de 5 a 6 metros de altura, aunque se encuentran ejemplares que llegan a medir hasta 14 metros. Tiene un tallo de 2-4 cm de diámetro aproximadamente, del cual se desprenden hojas con forma de abanico. Su base o parte inferior es dura y cubierta "cascas"; en el medio crecen las hojas de forma lanceolada de 2 a 3 metros aproximadamente, las cuales presentan un borde serrulado y filoso, el tallo termina en una gran inflorescencia o panícula, que puede alcanzar más de 1 metro de longitud, la cual es usada comúnmente como objeto de decoración (Suárez et al., 2009, p. 5135).

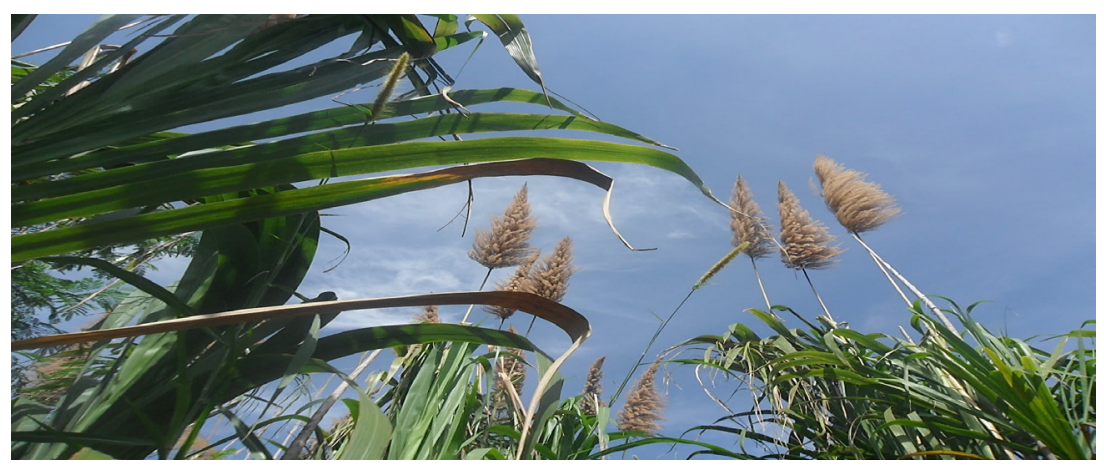

Figura 7. Gynerium sagittatum, conocida en Colombia como Caña Flecha Fuente: Los autores 
La reproducción de Gynerium sagittatum se realiza a través de los dos tipos: sexual y asexual. La menos común es la reproducción sexual; se realiza a través de las semillas que se agrupan en la inflorescencia en forma de panícula, y que producen 1.6 millones de semillas/kg (Francis, 2004, p. 370); las semillas germinan aproximadamente en 3 semanas, las plántulas crecen aproximadamente 20,30 y 50 centímetros, después de uno, dos y cuatro meses, respectivamente (Kalliola et al., 1992, p. 160). La reproducción asexual se realiza a través de rizomas, los cuales están constantemente activos en la producción de nuevas plantas en los espacios libres, en una distancia regular que oscila entre 15-20 centímetros de la planta principal (Kalliola et al., 1992, p. 160).

Se pueden identificar 2 variaciones de Gynerium sagittatum: la "grande", referida a aquellas con mayor altura, tallo grueso sin ramificaciones y de hojas anchas lanceoladas que crecen a ambos lados de tallo y se desdoblan en sus puntas; la "pequeña", por su tamaño menor, más esbelta, frecuentemente con tallos ramificados y hojas lanceoladas rígidas. También se puede identificar una tercera variación "intermedia" que presenta características comunes a los dos tipos principales (Kalliola et al., 1992, p. 158).

En Colombia se habla de la existencia de 3 tipos de Caña Flecha o Gynerium sagittatum, que pueden corresponder con las variedades explicadas anteriormente, pero su diferencia se determina de acuerdo con la calidad de la fibra que ofrece para la artesanía a saber: la "palma criolla" que da una sensación suave al tacto, permite un "ripiado" fino y es cultivada principalmente en los departamentos de Sucre y Córdoba; la "palma martinera", que se cultiva en Antioquia, y tiene una fibra rígida, quebradiza y de mayor tamaño. Además, no permite un "ripiado" fino sino más grueso; finalmente la "palma costera" que crece en zonas onduladas y a orillas del mar, presenta fibras quebradizas y de poca calidad para el trenzado.

Contreras et al. (1999) recomiendan su cultivo debido al potencial de celulosa con un uso potencial en la industria de papel; también por las posibilidades que ofrece como fuente para la fabricación de materiales substitutos de la madera, a partir de sus fibras, partículas y tiras. 
En Colombia su uso es asociado fuertemente con las tradiciones de la cultura indígena Zenú, quienes fueron una de las tribus de antiguos pobladores de la Costa Norte colombiana antes de la llegada de los españoles. Para los descendientes de estas comunidades, Gynerium sagittatum (Caña Flecha) es un material muy apreciado en la construcción de viviendas tradicionales con técnica mixta o "bahareque" (AGRA, 2016; Suárez et al., 2009 , p. 5135), y, especialmente, para la elaboración de diversos objetos artesanales, entre los cuales el más representativo es el llamado "sombrero vueltiao", símbolo de la artesanía colombiana en el mundo.

A pesar de ser muy reconocida como materia prima para la producción de artesanías Zenú, en Colombia existe muy poca información al respecto de esta especie. En el Departamento de Sucre, uno de los mayores productores de artesanías en Gynerium sagittatum, prácticamente no existe información consolidada con relación a su cultivo. Se habla de un promedio de 70 hectáreas cultivadas en el municipio de San Antonio de Palmito entre los años 2015 y 2018, con una producción promedio de 9,94 toneladas (Gobernación de Sucre, 2018), ver Tabla 18.

\section{Tabla 23}

Datos sobre Cultivos de Gynerium sagittatum en el Departamento de Sucre.

\begin{tabular}{cccccccc}
\hline Año & Cultivo & Período & Municipios & $\begin{array}{c}\text { Área } \\
\text { sembrada } \\
\text { (Has) }\end{array}$ & $\begin{array}{c}\text { Área } \\
\text { cosechada } \\
\text { (Has) }\end{array}$ & $\begin{array}{c}\text { Rendimiento } \\
(\mathbf{t} / \mathbf{h a})\end{array}$ & $\begin{array}{c}\text { Producción } \\
(\mathbf{t})\end{array}$ \\
\hline 2015 & $\begin{array}{c}\text { Caña } \\
\text { Flecha }\end{array}$ & Permanente & $\begin{array}{c}\text { San Antonio } \\
\text { de Palmito }\end{array}$ & 68 & 70 & 0,25 & 17,5 \\
\hline 2016 & $\begin{array}{c}\text { Caña } \\
\text { Flecha }\end{array}$ & Permanente & $\begin{array}{c}\text { San Antonio } \\
\text { de Palmito }\end{array}$ & 68 & 67 & 0,125 & 8,375 \\
\hline 2017 & $\begin{array}{c}\text { Caña } \\
\text { Flecha }\end{array}$ & Permanente & $\begin{array}{c}\text { San Antonio } \\
\text { de Palmito }\end{array}$ & 70 & 68 & 0,2 & 13,6 \\
\hline $\begin{array}{c}\text { Caña } \\
\text { Flecha }\end{array}$ & Permanente & $\begin{array}{c}\text { San Antonio } \\
\text { de Palmito }\end{array}$ & 74 & 70 & 21 & 0,3 \\
\hline
\end{tabular}

Fuente: Gobernación de Sucre (2018).

También se pueden encontrar iniciativas para la recuperación y manejo de especies vegetales asociadas a la producción artesanal en Sucre y Córdoba, entre las que se destaca el proyecto desarrollado por Carsucre y CVS, con el apoyo del ministerio del medio Ambiente y Fonade, para la recuperación y manejo de especies vegetales nativas medicinales y aptas 
para la producción artesanal en zonas indígenas Zenú de Córdoba y sucre (CARSUCRE, 2002).

El proyecto ejecutado entre octubre de 2001 y septiembre de 2002, se implementó con la iniciativa del establecimiento de 40 sub-proyectos agroecológicos de 5 hectáreas cada uno, las cuales se distribuirían así: 3 hectáreas de cultivo de Gynerium sagittatum; 1 hectárea con cultivo de plantas tintóreas y medicinales; 1 hectárea con árboles maderables, frutales y medicinales; generando un total de 120 hectáreas cultivadas con Gynerium sagittatum (CARSUCRE, 2002, p, 10).

Se destaca de esa iniciativa el interés por la preservación y disminución del impacto ambiental, de la mano de la recuperación de la cultura y los conocimientos ancestrales de las comunidades indígenas, la inversión de recurso humano y económico para la conservación de la biodiversidad y la cultura.

\section{Generalidades sobre Gynerium sagittatum}

En Martínez et al. (2018a) se hace una descripción detallada de las características generales de Gynerium sagittatum; se retoman aquí los elementos más importantes de esa descripción.

Las raíces son rizomas de los cuales surgen los tallos, también se desprenden de éstas algunas raíces laterales, las cuales son muy delgadas, débiles y flexibles con espesores variables alrededor de $1 \mathrm{~mm}$ o menos de diámetro (Francis, 2004, p. 370).

El tallo es cilíndrico, entre 2 y 4 centímetros de diámetro promedio. Crece de forma recta, aunque con algunas pequeñas alteraciones debido a las condiciones particulares en la que crezca la Planta, debido a esta condición se puede afirmar que el tallo no es completamente regular (ver Figura 13). En su parte inferior está recubierto con unas hojas que conforman una película de color café, la cual, una vez va alcanzando altura, pierde su color café, se vuelve cada vez más verde y surgen, a cada lado y de forma alternada, las hojas.

En los ejemplares adultos el tallo, justo después del grupo conformado por las hojas, reduce progresivamente su diámetro, para convertirse en un eje central que termina en un ápice que le da la forma de Flecha característica de su inflorescencia. 


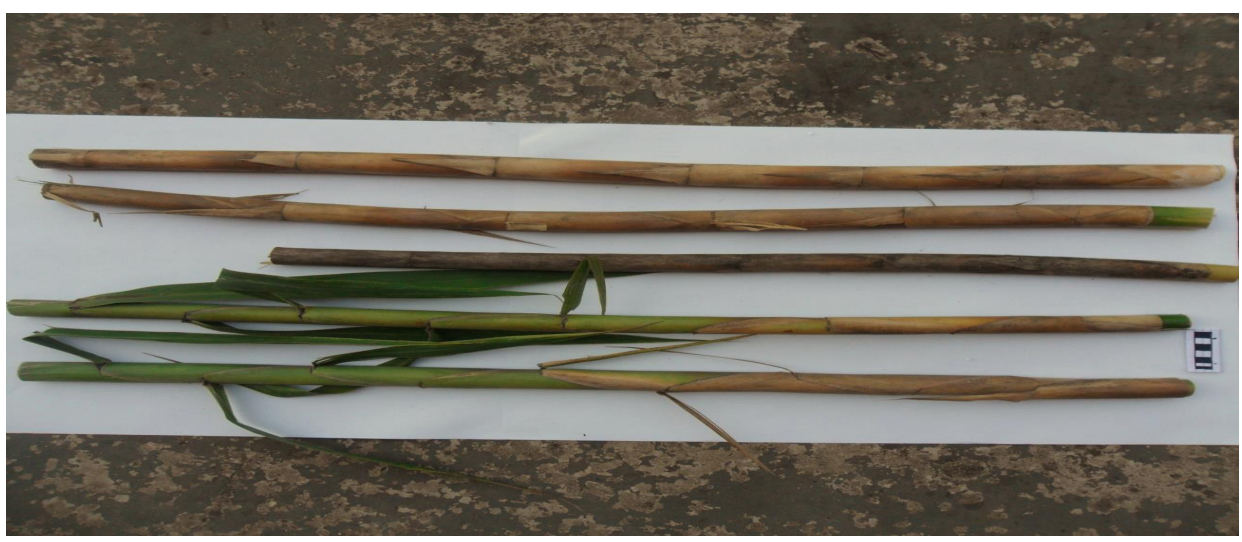

Figura 8. Tallo de Gynerium sagittatum

Fuente: Los autores

En su interior, el tallo está conformado por un tejido lignocelulósico que le da su rigidez, el cual, mientras la planta se encuentra recién cortada y verde, se constituye en un relleno completo del interior, muy similar a la caña de azúcar, pero una vez la planta ha sido cortada y comienza el proceso de secado, lentamente se empieza a contraer haciendo que el interior presente cavidades irregulares con presencia de tejido seco (ver Figura 11).

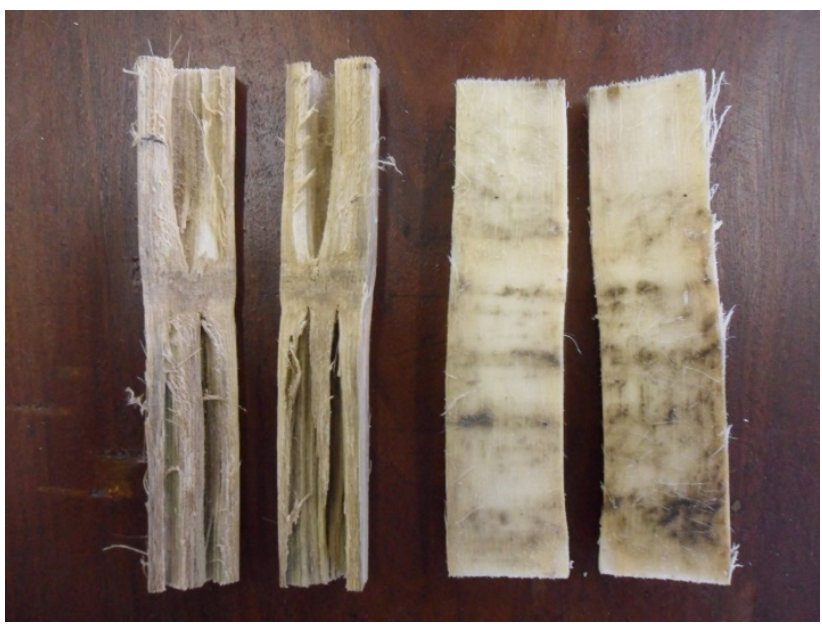

Figura 9. Tejido lignocelulósico de Gynerium sagittatum recién cortado y en proceso de secado

Fuente: Los autores 
Las hojas de Gynerium sagittatum son de forma lanceolada (1.70 2.0 mts.), de tipo uninervada, ya que presentan una sola nervadura central gruesa y un limbo o lámina de borde dentado o serrulado, lo que la vuelve áspera al tacto. La nervadura central es muy utilizada en Colombia por parte de los artesanos descendientes de la etnia Zenú, quienes la usan como materia prima de muchos tipos de artesanía elaborados con la trenza que se obtiene después de diferentes procesos de preparación.

Una de las características que distinguen a la especie Gynerium sagitattum, es la configuración en que crecen las hojas en grupo a cada lado del tallo. Las hojas son color verde oscuro y sin peciolo o de tipo sésil; nacen de forma decurrente, envolviendo al tallo alternadamente una tras otra, dejando en la parte inferior las hojas de menor tamaño y en la parte superior las de mayor tamaño entre 1.70-2.0 mts. Las hojas de mayor tamaño al ubicarse en la parte superior del grupo, se doblan producto de su propio peso y longitud, generando la forma agradable a la vista que es distintiva de su especie.

La inflorescencia de Gynerium sagittatum es otro de sus elementos distintivos; se conforma a partir de la prolongación del tallo, en la parte superior del grupo de hojas que forman el abanico. El tallo, al reducir su diámetro, conforma un eje central o raquis, del cual se desprende una panícula de color ocre amarillo semioscuro, que se va simplificando hacia el ápice, es decir reduce su tamaño y número de flores y ramas en la medida en que se acerca al final del eje central (ver Figura 15).

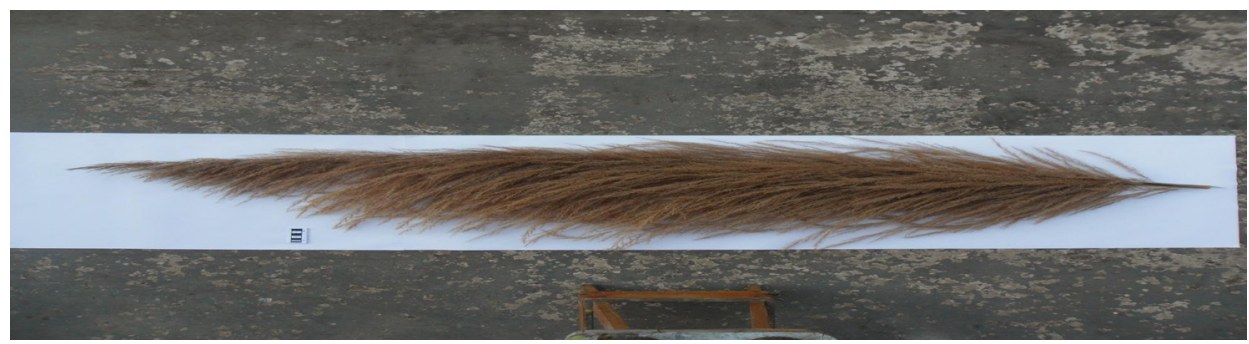

Figura 10. Inflorescencia característica de Gynerium sagittatum.

Fuente: Los autores

El conjunto del raquis central, junto con la panícula, conforma un elemento muy visible y característico de la especie, y puede medir en conjunto más de 2.0 metros de longitud. Los frutos que conforman la panícula son estrechos y oblongos, aproximadamente de $1 \mathrm{~mm}$ de largo, 
recubiertos de una especie de pelaje que facilita su dispersión a través del viento o el agua (Kalliola et al., 1992, p. 160).

\section{Investigaciones asociadas a las potencialidades Gynerium sagittatum como material alternativo para el desarrollo de nuevos productos forestales}

Martínez et al. (2018a) hacen una descripción del estado de la Investigación y Desarrollo con referencia a Gynerium sagittatum; se retoman aquí los elementos más importantes de esa descripción.

Durante los últimos 20 años se pueden identificar algunas investigaciones desarrolladas con relación a las posibilidades que ofrece Gynerium sagittatum, como material alternativo para el Desarrollo e Innovación de productos forestales, como sustituto al uso de la madera.

En primera instancia, es interesante la investigación desarrollada por Contreras e Owen de Contreras (1997), para la elaboración de un elemento estructural laminado, tipo parallam, con tiras de Gynerium sagittatum y adhesivo fenol-formaldehido. En la investigación de Contreras y Owen de Contreras (1997) se realiza un procesamiento mecánico de los colmos de Gynerium sagittatum, para obtener tiras con espesores promedios de 3 $\mathrm{mm}$, largo $3100 \mathrm{~mm}$ y ancho aproximado de $30 \mathrm{~mm}$. Los investigadores describen valores de ensayos menores a las exigencias de la norma que fue utilizada como referencia, observan una relación entre la calidad de la línea de cola y los valores bajos obtenidos, toman como causa negativa la mala humectación de la cola en las tiras cuando coinciden con la cara impermeable externa de Gynerium sagittatum (Contreras \& Owen de C., 1997 , p. 29).

Los autores manifiestan problemas en la elaboración de los elementos estructurales, debido a las grandes presiones internas que se observaron durante el proceso de construcción de las vigas.

El encofrado metálico falló a pesar de ser fabricado con elementos estructurales, sin poder soportar las grandes presiones internas, ya que se apreció en el transcurso del sometimiento a presión que todas sus caras se deformaron (reflejándose en las vigas una vez desencofradas), con el riesgo de colapsar la estructura del encofrado, además de la continua falla de los pernos que ejercían la presión constante. Por la razón descrita en el 
párrafo anterior, se pudo apreciar en toda la estructura física de las vigas; por consiguiente, en las probetas, que sus caras laterales presentaban un alto porcentaje de espacios vacíos (Contreras \& Owen de C., 1997, p. 33).

Entre las conclusiones que Contreras y Owen de Contreras (1997) exponen, se encuentra la sugerencia para desarrollar un estudio similar que elimine el factor negativo que observan en la cutícula impermeable exterior, además de plantear la necesidad de diseñar otro sistema de encofrado y de aplicación de presión en la elaboración de las vigas de forma que consolide mejor los elementos (p. 35).

Otra investigación fundamental se identifica en el trabajo de Contreras et al. (1999), en el cual proponen la elaboración de tableros aglomerados con partículas de Gynerium sagittatum y adhesivo urea-formaldehído. En esa investigación, los autores desarrollaron ensayos con tableros al 10 y $13 \%$ de resinosidad, con un mejor desempeño de aquellos con un 13\%; identifican debilidades en el proceso de producción de las partículas con las cuales elaboraron los tableros, relacionan la baja resistencia de los mismos a las características del molino y las cuchillas, que ocasionaron que gran parte de las partículas se convirtieran en polvillo, ocasionando posible falta de traba entre las partículas al elaborar los tableros (p. 133).

Puede atribuirse esta baja resistencia a que en los tableros elaborados bajo estos parámetros existió una mala calidad en la producción de las partículas con las cuales se hicieron los mismos, debido a la irregularidad de formas y tamaños obtenidos y analizadas de las probetas ensayadas las cuales se obtuvieron en el molino del laboratorio de pulpa y papel del LNPF, el cual está diseñado para la realización de astillas de bloques de madera sólida. Se pudo determinar que, por la mala calidad del afilado de las cuchillas, la gran velocidad de las revoluciones del portacuchillas ocasionó que se volvieran gran parte de las partículas en polvillo y otras de mediana e irregular tamaño ocasionando una posible falta de traba entre partículas al momento de elaborar los tableros. (Contreras et al., 1999, p. 133).

También identifican los autores debilidades en la composición física propia de Gynerium sagittatum; como factor determinante de la baja 
resistencia, explican que en su estructura anatómica la Caña Flecha presenta dos zonas bien definidas, una interna parenquimatosa, y otra externa esclerenquimatosa, que define la calidad del material lignocelulósico de la caña brava (Contreras et al., 1999).

Contreras et al. (1999) concluyen que los tableros desarrollados pueden ser aplicados a múltiples usos, ya que presentan buena consistencia y una apariencia agradable. Recomiendan la aplicación de tratamientos contra la humedad y la prevención de agentes xilófagos para su posterior producción industrial (p. 129).

En otro trabajo desarrollado por el mismo grupo de Investigadores (Contreras et al., 2008), se da continuidad al trabajo y se determinan los niveles de sostenibilidad en el proceso de fabricación de los tableros con Gynerium sagittatum, a partir de un método propio que desarrollaron denominado "método ACV-Coclowen". Identifican los indicadores negativos más significativos en las etapas de adquisición del adhesivo utilizado, la transformación de las cañas en partículas, tamizado y clasificación de partículas, secado de partículas, encolado, la conformación del tablero a través de presión/calor, y el dimensionamiento final del tablero (p.57).

Los autores sugieren en sus conclusiones hacer uso de adhesivos más amigables con el medio ambiente, aplicar normas de seguridad industrial en la etapa de encolado, para disminuir los riesgos de toxicidad al operario; para el diseño de la industria, utilizar residuos de madera para alimentar las calderas en el proceso de secado de partículas (Contreras et al. 2008, p. 58).

Una investigación más reciente, desarrollada por Gallego et al. (2014), Diossa et al. (2017) (a partir de Gallego, 2014), propone el desarrollo de tableros aglomerados de Gynerium sagittatum, sin uso de adhesivos sintéticos. Explican los usos potenciales de la lignina presente en Gynerium sagittatum para reemplazar los adhesivos en tableros aglomerados, proponen procesos de pretratamiento como el steam explosion, para el desarrollo de tableros de partículas de alta densidad de Gynerium sagittatum prensadas sin uso de aditivos. Identifican una relación entre los mejores resultados a las propiedades mecánicas, y la severidad del pretratamiento con steam explosión (Gallego et al., 2014, p. 110). 
De la investigación de Gallego et al. (2014), se observa como aspecto positivo la reducción a cero en el uso de adhesivos, pero se considera importante analizar la relación del consumo energético que implica la elaboración del producto a través del pretratamiento y el posterior proceso de prensado con calor.

En las investigaciones referenciadas, es posible identificar aspectos aún por mejorar con relación a la sostenibilidad de los procesos de producción de productos forestales alternativos con Gynerium sagittatum. Aspectos relativos a la superación de las debilidades provenientes de las características físicas inherentes al material, la transformación de los colmos, la conformación de los elementos sean tableros o elementos estructurales, las características de diseño de la planta industrial, el tipo de adhesivos utilizados y el tipo de recursos energéticos utilizados en el proceso de producción, son, entre otros, elementos a problematizar en la búsqueda de Innovación desde el Life Cycle Design y el Design para la sustentabilidad.

\section{Conocimientos de las comunidades indígenas Zenú asociados al procesamiento de Gynerium sagittatum}

Martínez et al. (2018a) hacen una recopilación de los aspectos más importantes con relación a las técnicas artesanales indígenas para la producción de artesanías con Gynerium sagittatum, las cuales se encuentran registradas en la norma NTC 5714 (Icontec, 2009). Se presentan a continuación los elementos más destacados de esa selección.

Uno de los documentos que recoge parte del conocimiento indígena Zenú con relación al procesamiento de la Caña Flecha, es la Norma Técnica Colombiana NTC 5714 (Icontec, 2009), en la cual se compilan una serie de procesos que hacen parte de la cadena productiva de las artesanías elaboradas en fibras de Caña Flecha; con la finalidad de establecer unos criterios de sostenibilidad para la utilización de un sello ambiental colombiano para esos productos, y, de esa forma, promover la oferta y demanda de productos que generen un menor impacto en el ambiente, como herramienta de comunicación que diferencie aquellos productos que presenten un mejor desempeño ambiental (Icontec, 2009, p. i).

En la norma NTC 5714 se establecen criterios ambientales, que cubren prácticamente todo el proceso de producción artesanal, los cuales van desde el cultivo de la Caña Flecha hasta la confección y elaboración 
de los productos artesanales, e incluye el rotulado del producto. Entre los procesos de los que habla la norma, se destacan aquellos que hacen parte del conocimiento ancestral de las comunidades indígenas Zenú: despaje y desvarite, raspado, blanqueado, ripiado, tinturado y trenzado de las fibras de Caña Flecha (Icontec, 2009, p. 8-15).

El "despaje y desvarite" consiste en retirar la parte ancha de la hoja dejando solo la nervadura central como parte aprovechable para la elaboración de artesanías. Este proceso se realiza con herramientas afiladas, además de exigir su realización con elementos mínimos de seguridad, limpieza personal y disposición de residuos, los cuales pueden aprovecharse como alimento para animales o abonos orgánicos (Icontec, 2009, p. 8).

El "raspado" consiste en el proceso de retirar la capa vegetal de la nervadura central que ha sido seleccionada a partir del "desvarite":

Proceso en el cual se retira la capa vegetal de la vena de la hoja, empleando un cuchillo de filo medio. El procedimiento se realiza colocando la zapatilla en la pierna, sobre la cual se coloca la fibra y con el cuchillo se hace el raspado de las hojas, este procedimiento se repite cuantas veces sea necesario, hasta que la fibra quede completamente suave. Este proceso es determinante para la calidad de la fibra. (Icontec, 2009, p.9).

El proceso de "blanqueado" como su nombre lo indica consiste en dar un tono más claro a las fibras de Caña Flecha para su posterior uso:

Se realiza en agua de caña agria. Para ello se dispone suficiente agua limpia en un recipiente según la cantidad de fibra a blanquear. Se pela la caña agria, quitándole la concha a la vara con un machete. Se machaca, pila o tritura la caña pelada sobre una superficie dura para que suelte el jugo y luego se exprime este en el agua. Se revuelve el agua se depositan los manojos de Caña Flecha a blanquear, poniendo encima el bagazo de la caña agria para mantenerla sumergida adicionarle ácido cítrico, dejándola en el agua de caña agria durante 12 h o una noche.

Al día siguiente se saca la Caña Flecha, se lava bien con la misma agua, evitando que se adhieran pedazos del bagazo, se pone al sol y sereno durante $2 \mathrm{~d}$ o $3 \mathrm{~d}$, hasta que adquiera su color natural. Hay que evitar sobre exponerla al sol, ya que se vuelve 
amarilla o se ensucia con el polvo. En caso de que falte caña agria, se puede usar naranja o limón (Icontec, 2009, p.11).

Es interesante de este proceso la utilización de otras especies vegetales para realizar el blanqueamiento, así como la reutilización del agua para la repetir la misma actividad y para el riego de las plantas.

El proceso de "ripiado" consiste en dividir la fibra en fragmentos más delgados, lo cual condicionará el tipo de calidad del trenzado posterior:

Se hacen fibras delgadas o "pencas" de acuerdo con la calidad de la trenza que se vaya a tejer. Es importante ripiar todo el lote de la Caña Flecha que se está preparando de una sola vez, ya que pueden quedar pencas de diferente grosor el trenzado no quedará uniforme (Icontec, 2009, p.12).

El proceso de "tinturado" de la fibra inicia con la escogencia del barro, consiste en aplicar unas tonalidades diferentes a la fibra a través de la utilización de pigmentos vegetales que se adicionan al barro. El tono más tradicional que se obtiene es el negro, pero se pueden conseguir diferentes colores a partir de la utilización de variadas especies como pigmentos vegetales:

Se escoge barro de color negro intenso que no presente sedimentos. Este barro es un barro que puede provenir de pozos. Las características del barro adecuado es que no esté pisado o "trajinado", que sea de color negro, que no presente arena y no tengan vetas (que no tenga otro tipo de color que no sea negro). El tiempo de duración del barro es de aproximadamente de 6 meses. Se requiere tener un recipiente de uso exclusivo para el barro, el cual se le añade bija, conchas de plátano y otros componentes de tipo vegetal (Icontec, 2009, p.13).

Se introduce la fibra dentro del barro por $24 \mathrm{~h}$, se saca y cocina durante 2 h con plantas tintóreas, siendo la más utilizada la Bija o Limpiadientes; el proceso se repite dos o tres veces para obtener un buen color negro. La fibra no puede durar más de 3 días en el barro, porque se puede deteriorar la fibra. El tinturado es ideal hacerlo sobre la fibra ripiada, pero en muchos casos se hace sobre la trenza ya tejida (Icontec, 2009, p.14). 
Linares et al. (2008), describen el proceso de "tinturado" de las fibras de Caña Flecha, especifican las especies vegetales más utilizadas en el proceso de teñido, entre las que destacan: Cañagria (cogollos de Costus laevis), limón (Citrus limon), naranja agria (Citrus aurantium), dividivi (Caesalpinia coriacea), jagua (Genipa americana) y achiote (Bixa orellana), con hojas de bija (Arrabidaea chica) y cáscara o bacota de plátano (Musa $X$ balbisiana). Explican también que el barro es seleccionado de terrenos sedimentarios ricos en sustancias alcalinas (pH 8 a 9) (Linares et al., 2008).

El "trenzado" constituye una de las prácticas más tradicionales de las comunidades indígenas Zenú. Se empieza en esta actividad desde temprana edad y se mantiene a lo largo de los años; transmiten este conocimiento ancestral de generación en generación:

La trenza se forma con un número impar de conjuntos de pencas blancas y negras llamadas "pares" o "pies". Esta trama es fundamental para identificar y denominar las diferentes trenzas y calidades de sombrero: 7 pares, "Lica"; de 11, "Acotilla"; de 15, "Quinciana"; de 19, 21, 23, de 27, siendo más fina la de mayor número de pares de pencas (Icontec, 2009, p.15).

Las diferentes técnicas artesanales utilizadas en el procesamiento de la Caña Flecha, representan un conocimiento ancestral que ha perdurado a través del tiempo, el cual posee un fuerte contenido simbólico y cultural, que se caracteriza por ser una producción armoniosa con el contexto en el que surgió. Son técnicas que pueden ser reinterpretadas en el campo del Design para la sustentabilidad, en busca de generar productos con un alto sentido de Identidad e Innovación, diferenciándose en el mercado por su capacidad de establecer procesos más sustentables y amigables con el contexto natural y cultural.

\section{La Investigación sobre las técnicas tradicionales indígenas para el desarrollo de productos sostenibles con Gynerium sagittatum}

En la actualidad se desarrolla una investigación dentro del Doctorado en Design de la Universidade Estadual Paulísta, UNESP. Esta investigación tiene por nombre "Técnicas tradicionais indígenas para o desenvolvimento de produtos de Design sustentável com Gynerium sagittatum". Se describen a continuación los aspectos más importantes de los métodos desarrollados en ese estudio en curso (Martínez et al., 2018b; Martínez et al., 2018c). 
La investigación consiste en un estudio experimental y proyectual, desarrollado en tres fases, a saber: Fase 1 Descriptiva-Exploratoria. En esta fase se realizó el conocimiento del material, tomando contacto con el mismo, a partir de actividades exploratorias realizadas en las dependencias del Laboratorio Didáctico de Materiales y Prototipos (LDMP) de la UNESP, Bauru, teniendo como base diferentes procesos artesanales desarrollados por los indígenas Zenú de Colombia y aplicados al procesamiento de los colmos, siendo estos el beneficiamiento manual, el trenzado y el teñido, con la finalidad de establecer alternativas para el desarrollo de materiales tipo paneles, usando conceptos de sustentabilidad para la producción de objetos de diseño. El trabajo fue desarrollado en asociación con la comunidad indígena Zenú asentada en la aldea "Villa Rosita" en Sincelejo, Sucre, Colombia, con la finalidad de observar y aprehender las técnicas a ser aplicadas en diferentes desarrollos.

Fase 2 de Tipo Experimental. En esta etapa, de abordaje cuantitativo, se mejoraron los desarrollos apuntados en la primera fase y se sometieron a ensayos de caracterización física y mecánica dos clases de materiales tipo paneles desarrollados usando una reinterpretación de las técnicas tradicionales de los indígenas Zenú.

Los dos tipos de materiales desarrollados fueron sometidos a una prueba preliminar para conocer su comportamiento (ver Figura 16).

La elaboración de las probetas para el ensayo preliminar se realizó en el laboratorio (LDMP) de la UNESP, adoptando las especificaciones de la norma técnica de ensayos aplicados a los paneles de madera aglomerada NBR14810-2 (ABNT, 2013).

Las pruebas para establecer la caracterización física y mecánica de los materiales en el ensayo preliminar fueron: densidad, humedad, absorción de agua, tracción perpendicular, tracción paralela, flexión estática, compresión longitudinal. Estos ensayos se realizaron en una máquina universal de ensayo DL 30.000 marca EMIC, propiedad de la Facultad de Ingeniería, FEB, UNESP, campus Bauru, Brasil. 


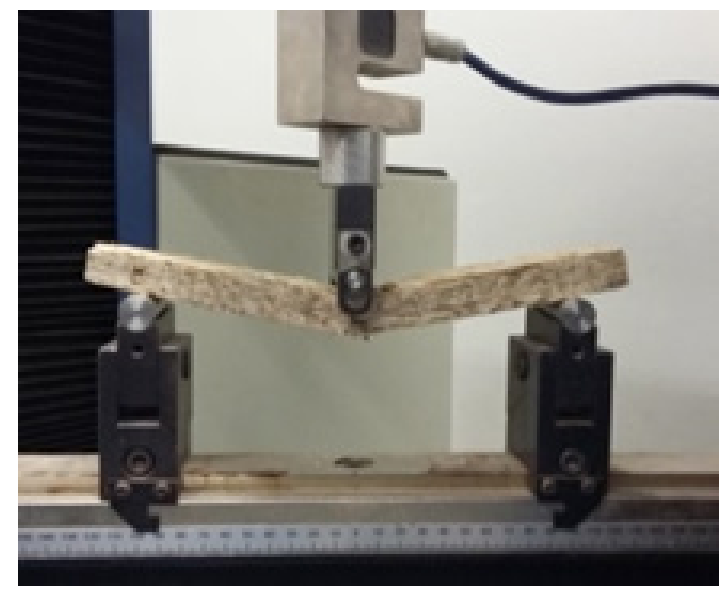

Figura 11. Probeta extraída de panel de partículas de Gynerium sagittatum, sometida al ensayo de flexión estática.

Fuente: Los autores

A partir del ensayo preliminar, se determinó la poca resistencia del material de partículas aglomeradas de Gynerium sagittatum elaborado con procesos artesanales, por el cual se decidió continuar el proceso experimental con el otro material desarrollado.

Para el análisis se utilizó software SSPS Statistic 23 para el procesamiento de la información. Se realizaron análisis de las características físicas y mecánicas del material y contrastando todos los datos de los materiales probados.

Fase 3 de Tipo Proyectual. Corresponde aquí a la Etapa de Diseño de producto de mobiliario, utilizando los materiales desarrollados con base en Gynerium Sagittatum. Se diseñó un prototipo de mobiliario conceptual para interiores utilizando chapas del material desarrollado a partir de Gynerium sagittatum con la finalidad de verificar su aplicabilidad. En esta etapa de la investigación, se construyeron modelos a partir del proceso de diseño. 


\section{Conclusiones}

Como conclusiones parciales de la investigación en curso se pueden destacar entre otras:

- Los conocimientos ancestrales, las técnicas y procesos para la producción de artesanías por parte de las comunidades indígenas de Colombia, en este caso los saberes que sobreviven en la comunidad Zenú, representan un gran insumo con mucho potencial para su reinterpretación y aplicación en diferentes procesos Innovación para la producción de materiales substitutos de la madera utilizando conceptos de sostenibilidad.

- Se consiguió evidencia en este estudio, sobre el hecho de encontrar aspectos positivos en los procesos artesanales aplicados a la obtención de partículas de Gynerium sagittatum.

- Algunas de las técnicas artesanales aplicadas a la producción de partículas de Gynerium sagittatum, permiten superar aspectos negativos referenciados en investigaciones precedentes sobre materiales substitutos de la madera con Gynerum sagittatum, desarrolladas por Contreras y Owen de C., (1997) y Contreras et al, (1999).

- En esta investigación fue posible innovar y desarrollar materiales sustitutos de la madera, utilizando conceptos de sostenibilidad, a partir de Gynerium sagittatum.

- Fue posible aplicar nuevos materiales con base en Gynerium sagittatum, en el diseño y producción de mobiliario utilizando conceptos de sostenibilidad.

- Algunos procesos de producción de materiales aglomerados no fueron posibles de ser desarrollados mediante procesos más artesanales, debido a la importancia de garantizar aspectos de control de calidad para obtener un mejor desempeño de los materiales desarrollados. 


\section{Referencias}

ABNT (2013). Painéis de partículas de média densidade. Parte 2: Requisitos e métodos de ensaio. ABNT - Associação Brasileira de Normas Técnicas.

AGRA. (2016). Técnicas vernáculas. Programa Paisajes de Conservación. USAID, MINAMBIENTE, Fondo Patrimonio Natural, AGRA Arquitectos (Anzellini García Reyes - Arquitectos). https://www.rds.org. co/es/recursos/tecnicas-vernaculas

Corporación Autónoma Regional de Sucre [CARSUCRE]. (2002). Recuperación y manejo de especies vegetales nativas medicinales y aptas para la producción artesanal en zonas indígenas Zenúes de Córdoba y Sucre. Corporación autónoma regional de los valles del Sinú y del San Jorge, C.V.S. Corporación autónoma regional de Sucre, CARSUCRE, Ministerio del Medio Ambiente, Fondo Financiero de Proyectos de Desarrollo, FONADE.

Contreras, W., Cloquell, B., \& Owen de C., M. (2008). Determinacion de los niveles de sostenibilidad del proceso de fabricacion de tableros de cana brava (Gynerium sagittatum), a partir del metodo ACV-Coclowen. La Revista Forestal Venezolana, 52(1), 47-60. http://www. saber.ula.ve/bitstream/handle/123456789/29085/articulo5.pdf?sequence $=1$ \&isAllowed $=\mathrm{y}$

Contreras, W., \& Owen de C., M. (1997). Elaboración De Un Elemento Estructural Laminado, Tipo Parallam, Con Tiras De Caña Brava Gynerium Sagittatum Y Adhesivo Fenol-Formaldehido. Revista Forestal Venezolana, 41(1): 29-36. http://www.saber.ula.ve/bitstream/ handle/123456789/24264/articulo41_1_3.pdf?sequence=1

Contreras, W., Owen de C., M.E., Garay, D.A., \& Contreras, Y. (1999). Elaboración de tableros aglomerados de partículas de Caña Brava (Gynerium sagittatum) y adhesivo urea-formaldehído. Revista Forestal Venezolana. 43(2), 129-135. http://www.saber.ula.ve/bitstream/handle/123456789/24194/articulo43_2_1.pdf;jsessionid=5F3316C3AAF3EFF602509F8A2B7CA770? sequence $=1$

Coradin, L., Siminski, A., \& Reis, A. (Eds.). (2011). Espécies Nativas da Flora Brasileira de Valor Econômico Atual ou Potencial. Plantas para o Futuro - Região Sul. Ministério do Meio Ambiente. 
Diossa, G. G., Velásquez, J. A., Quintana, G. C., \& Gómez, V. (2017). Efecto de la presión de prensado y la adición de lignina kraft en la producción de tableros aglomerados auto enlazados a partir de Gynerium sagittatum pre tratada con vapor. Maderas. Ciencia y tecnología, 19(4), 525-538. https://doi.org/10.4067/S0718221X2017005001201

Durst, P., Killmann, W., \& Brown, C. (2004). Asia's new woods. Journal of forestry, 102(4), 46-53. https://doi.org/10.1093/jof/102.4.46

Francis, J. (ed.). (2004). Wildland Shrubs of the United States and Its Territories: Thamnic Descriptions: Volume 1. International Institute of Tropical Forestry, Department of Agriculture, Forest Service, Rocky Mountain Research Station.

Gallego, G. (2014). Tableros sin aditivos a partir de Caña Flecha (Gynerium sagittatum). [Trabajo de pregrado, Universidad Pontificia Bolivariana]. Repositorio UPB. https://repository.upb.edu.co/bitstream/ handle/20.500.11912/3148/Tableros\%20sin\%20aditivos\%20a\%20 partir\%20de\%20la\%20ca\%C3\%Bla\%20flecha.pdf?sequence=1\&isAllowed=y

Gallego, G., Velásquez, J., \& Quintana, G. (2014). Tableros sin aditivos a partir de Gynerium sagittatum. Revista investigaciones aplicadas, 8(2), 101-112. https://dialnet.unirioja.es/servlet/articulo?codigo $=5001548$

Gobernación de Sucre (2018). Datos estadísticos del Sector Agropecuario cultivo de Caña Flecha en Sucre, durante los años 2015 al 2018. Secretaría de Desarrollo Económico y Medio Ambiente de Sucre.

ICONTEC. (2009). Norma Técnica Colombiana, NTC 5714. Sello Ambiental Colombiano. Criterios Ambientales para Artesanías, Sombreros $y$ otros productos del diseño, elaborados en fibras de Cañaflecha con Tecnología Artesanal. Instituto Colombiano de Normas Técnicas (ICONTEC). http://www.minambiente.gov.co/images/normativa/ Otros/NTC/2009/NTC_5714_2009.pdf

Kalliola, R., Puhakka, M., \& Salo, J. (1992). Intraspecific variation, and the distribution and ecology of Gynerium sagittatum (Poaceae) in the western Amazon. Flora, 186(3-4), 153-167. https://doi. org/10.1016/S0367-2530(17)30531-5 
Linares, E., Galeano, G., García, N., \& Figueroa, Y. (2008). Fibras vegetales utilizadas en artesanías en Colombia. Artesanías de Colombia S.A., Ministerio de Comercio, Industria y Turismo. Instituto de Ciencias Naturales, Facultad de Ciencias - Universidad Nacional de Colombia.

Manzini, E., \& Vezzoli, C. (2002). O desenvolvimento de produtos sustentáveis. Os requisitos ambientais dos produtos industriais. São Paulo, Brasil. Editora da Universidade de São Paulo, 368 p.

Martínez, P., Da Cruz, L. P., \& Barata, T. (2018a). Conhecimento indígena e processos para o desenvolvimento de produtos de design sustentável com Cana-Flecha (Gynerium Sagittatum). En A. Arruda, P. Machado, L. Liberlotto, Design, Artefatos e Sistema Sustentável (pp. 247-266). Blucher. https://doi.org/10.5151/9788580392982-12

Martínez, P., Da Cruz, L. P., \& Barata, T. (2018b). Procesos artesanales para la producción sostenible de tableros de Caña Flecha (Gynerium sagittatum). Mix sustentável, 4(2), 59- 70. https://doi. org/10.29183/2447-3073.MIX2018.v4.n2.59-70

Martínez, P., Da Cruz, L. P., \& Barata, T. (2018c). Reinterpretação de técnicas tradicionais indígenas para o desenvolvimento de produtos de design sustentável. En R. Cardoso, P. Landim, M. Gurgel, F. Orsi, Interdisciplinaridade nas pesquisas em design, arquitetura e urbanismo. (pp. 25-35). Canal 6.

Pereira, M., \& Beraldo, A. (2008). Bambu de corpo e alma. Canal6.

Suárez, I. E., Araméndiz, H., \& Pastrana, I. J. (2009). Micro propagación de Caña Flecha (Gynerium sagitatum Aubl.). Revista Facultad Nacional Agronomía de Medellín, 62(2), 5135-5143. https://revistas.unal. edu.co/index.php/refame/article/view/24925 